\title{
Modelo lógico e matriz de critérios para avaliação da assistência à pessoa com transtorno mental em conflito com a lei
}

\author{
Logical model and matrix of criteria for assessing care to people \\ with mental disorders in conflict with the law
}

Lannuzya Veríssimo e Oliveira (https://orcid.org/0000-0002-6881-898X) ${ }^{1}$

Ardigleusa Alves Coelho (https://orcid.org/0000-0001-8869-3793) ${ }^{2}$

Severina Alice da Costa Uchôa (https://orcid.org/0000-0002-2531-9937) ${ }^{1}$

Pétala Tuani Cândido de Oliveira Salvador (https://orcid.org/0000-0002-3208-6270) ${ }^{1}$

Cláudia Helena Soares de Morais Freitas (https://orcid.org/0000-0003-0265-5396) ${ }^{3}$

${ }^{1}$ Universidade Federal do Rio Grande do Norte. Av. Sen. Salgado Filho 3000 Lagoa Nova. 59064-720 Natal RN Brasil.

lannuzya@ufrn.edu.br

${ }^{2}$ Universidade Estadual da Paraíba. Campina Grande PB Brasil.

${ }^{3}$ Universidade Federal da Paraíba. João Pessoa PB Brasil.

\begin{abstract}
We aim to describe the construction and validation of the logical model and the matrix of criteria for assessing care to people with mental disorders in conflict with the law. This is a methodological research, carried out from February to December 2019, following three procedures: 1) theoretical - composed of an evaluability study performed from documentary research and interviews with key informants; 2) empirical - construction of the collection instrument and selection of experts for the validation process of the proposed evaluation instrument; and 3) analytical - performing two Delphi steps. The evaluability study resulted in the elaboration and agreement of the logical model; the analysis and comparison between the reality of the policy and the logical model; and the elaboration of the matrix of criteria. The criteria matrix content adequacy was assessed by 16 experts in the Delphi 1 step and 12 experts in the Delphi 2 step, whose content was validated with a Content Validity Coefficient of 0.93. The logical model and the matrix of criteria proposed in this study are expected to direct health professionals, researchers, workers, and other social actors to assess this clientele's care.
\end{abstract}

Key words Evaluation of health programs and projects, Evaluation of research programs and tools, Validation study, Mental disorders, Inmates
Resumo Objetiva-se descrever a construção e validação do modelo lógico e da matriz de critérios para avaliação da assistência à pessoa com transtorno mental em conflito com a lei. Trata-se de pesquisa metodológica, desenvolvida de fevereiro a dezembro de 2019, seguindo três procedimentos: 1) teóricos - composto por estudo de avaliabilidade, realizado a partir de pesquisa documental e entrevista com informantes-chave; 2) empíricos - construção do instrumento de coleta e seleção de experts para o processo de validação do instrumento de avaliação proposto; e 3) analíticos - realização de duas etapas Delphi. O estudo de avaliabilidade resultou na elaboração e pactuação do modelo lógico; na análise e comparação entre a realidade da política e o modelo lógico; e na elaboração da matriz de critérios. A adequabilidade do conteúdo da matriz de critérios foi avaliada por 16 experts na etapa Delphi 1 e por 12 na etapa Delphi 2, a qual teve seu conteúdo validado com Coeficiente de Validade de Conteúdo de 0,93. Espera-se que o modelo lógico e a matriz de critérios propostos neste estudo direcionem profissionais de saúde, pesquisadores, trabalhadores e demais atores sociais para a avaliação da assistência a esta clientela.

Palavras-chave Avaliação de programas e projetos de saúde, Avaliação de Programas e Instrumentos de Pesquisa, Estudo de validação, Transtornos mentais, Prisioneiros 


\section{Introdução}

No Brasil, os direitos à dignidade, à autonomia e à inserção social das pessoas com transtorno mental e a gradual desinstitucionalização dos usuários de saúde mental, bem como a proibição de internação prolongada em instituições asilares ou com características análogas a estas, foram estabelecidas na Lei $n^{\circ}$ 10.216/2001 - marco da Reforma Psiquiátrica ${ }^{1}$.

Assim, a internação da pessoa com transtorno mental passou a ser indicada somente quando o tratamento fora do hospital se mostrar ineficaz. São previstas três possibilidades: a internação voluntária, com o consentimento do paciente; a involuntária, sem consentimento e a pedido de outra pessoa; e a compulsória, que é determinada pela justiça².

Inserem-se na modalidade de internação compulsóriaas pessoas com transtorno mental autoras de delitos. Nesses casos, são aplicadas as Medidas de Segurança estabelecidas pelos artigos 96 a 99 do Código Penal $(\mathrm{CP})$ brasileiro àqueles considerados inimputáveis ou semi-imputáveis, os quais são incapazes de entender a ilicitude de seus atos e têm indicação de internação em Hospital de Custódia e Tratamento Psiquiátrico (HCTP) ou estão sujeitos a tratamento ambulatorial ${ }^{3}$.

Contudo, o que se observa é um descompasso entre a Política Nacional de Saúde Mental vigente, publicada a partir da Lei $n^{\circ} 10.216 / 2001$, e a aplicação das Medidas de Segurança, uma vez que quesitos jurídicos centrados na capacidade de entendimento e na autodeterminação na execução do ato ilícito, combinados à avaliação pontual e exclusiva do médico psiquiatra, culminam com o encarceramento, por vezes perpétuo, do "louco infrator" nos HCTPs ${ }^{3,4}$.

De acordo com o último levantamento realizado pelo Departamento Penitenciário Nacional, em 2011 existiam no Brasil 26 Estabelecimentos de Custódia e Tratamento Psiquiátrico (ECTP), a saber: 23 HCTPs e três Alas de Tratamento Psiquiátrico (ATPs) $)^{5}$. Em 2014, segundo pesquisa realizada pelo Sistema Geopresídios do Conselho Nacional de Justiça (CNJ), existiam 35 espaços de caráter asilar para confinamento de pessoas com transtorno mental em conflito com a lei no Bra$s i l^{6}$. É válido considerar que tais estabelecimentos permanecem ligados aos sistemas de segurança e, apesar de serem chamados "hospitais", foram historicamente regidos segundo os princípios da execução penal a partir da Lei no 7.210 , de $1984^{7}$.

Tais espaços apresentavam as seguintes características: ausência de Projeto Terapêutico
Singular (PTS); modelo de contenção determinado pela legislação criminal em detrimento das políticas de saúde; escassa participação das redes de saúde e de assistência social; tratamentos inadequados, insuficientes ou inexistentes; reforço do estigma, do preconceito social e eternização da institucionalização dos pacientes; perda, quase sempre irreversível, dos vínculos familiares e sociais; e consumo impróprio dos recursos públicos ${ }^{8}$.

Atentos a tais demandas, os Ministérios da Justiça e da Saúde têm implementado ações que visam à melhoria da assistência à saúde mental ofertada às pessoas em conflito com a lei, a exemplo da Resolução no 113, do Conselho Nacional de Justiça, que, em abril de 2010, incluiu a Lei da Reforma Psiquiátrica no regimento do processo de aplicação da Medida de Segurança, juntamente com a Lei da Execução Penal ${ }^{9}$.

Ainda em 2010, o Conselho Nacional de Política Criminal e Penitenciária, na Resolução $\mathrm{n}^{\circ} 4$, destacou a necessidade da observação dos princípios estabelecidos pela Lei no 10.216/2001 na execução da Medida de Segurança e propôs orientações importantes, como a abordagem intersetorial, o acompanhamento psicossocial contínuo, a individualização da medida imposta, com respeito às singularidades psíquicas, sociais e biológicas de cada um, a inserção social, entre outros $^{10}$.

Mais recentemente, em 2014, o Ministério da Saúde, através da Portaria no 94, em consonância com a Política Nacional de Atenção Integral à Saúde das Pessoas Privadas de Liberdade (PNAISP), instituiu o serviço de avaliação e acompanhamento de medidas terapêuticas aplicáveis à pessoa com transtorno mental em conflito com a lei, no âmbito do Sistema Único de Saúde (SUS) ${ }^{11}$.

Este estudo justifica-se pela escassez na literatura, sobretudo nas ciências da saúde, de estudos que avaliem a assistência às pessoas com transtornos mentais em conflito com a lei no contexto brasileiro; pela limitação de investimentos em pesquisas avaliativas de programas e políticas de saúde, associada à dificuldade de avaliar uma intervenção complexa, com caráter multidimensional e que envolve serviços, sistemas e múltiplas atividades; e ainda pela inexistência de um instrumento para orientar a avaliação da assistência à saúde mental às pessoas em conflito com a lei de modo contínuo e sistemático e que produza mudanças na produção do cuidado a esse grupo.

Nessa perspectiva, objetiva-se descrever o processo de construção e validação domodelo 
Trata-se de pesquisa metodológica, com abordagem mista, desenvolvida de fevereiro a dezembro de 2019, a partir do referencial metodológico adaptado da Psicometria de Pasquali ${ }^{12}$, baseada nas três etapas: 1) procedimentos teóricos, para identificar os conteúdos que compuseram o modelo lógico e a matriz de critérios avaliativos; 2) procedimentos empíricos, quando a validação de conteúdo da matriz de critérios com experts na área foi delineada; e 3) procedimentos analíticos, quando a validação foi analisada, por meio da técnica Delphi, de modo a confirmar ou refutar a validação da matriz de critérios proposta.

Quanto aos procedimentos teóricos, com a finalidade de construir o modelo lógico e a matriz de critérios avaliativos, realizou-se um estudo de avaliabilidade com abordagem qualitativa, o qual precede a etapa de avaliação propriamente dita, pois permite saber em que medida um programa/política está em condições de ser submetido a uma avaliação ${ }^{13}$.

É um processo cíclico e dinâmico que segue as etapas: I) esclarecimentos sobre os objetivos e metas do programa/política; II) desenvolvimento e pactuação do modelo lógico com os interessados; III) análise e comparação entre a realidade do programa/política e o modelo lógico; IV) elaboração de recomendações para realização da avaliaçãodo programa/política ${ }^{13}$.

Inicialmente, com objetivo de elencar dados relevantes e estabelecer apropriações sobre a PNAISP no que concerne à assistência em saúde mental das pessoas em conflito com a lei, foi realizada uma leitura dos seguintes documentos: Portaria Interministerial $n^{\circ} 1$, de 2014, que institui a PNAISP no âmbito do SUS ${ }^{14}$; Portaria no 94/ GM/MS, de 2014, que institui o serviço de avaliação e acompanhamento de medidas terapêuticas aplicáveis à pessoa com transtorno mental em conflito com a lei, no âmbito do SUS ${ }^{11}$; Lei $n^{\circ} 10.216$, de 2001, que dispõe sobre a proteção e os direitos das pessoas portadoras de transtornos mentais e redireciona o modelo assistencial em saúde mental ${ }^{2}$; Portaria no 95 , de 14 de janeiro de 2014, que dispõe sobre o financiamento do serviço de avaliação e acompanhamento às medidas terapêuticas aplicáveis ao paciente judiciário, no âmbito do Sistema Único de Saúde (SUS) ${ }^{15}$; e
Portaria no 142, de 28 de fevereiro de 2014, que estabelece normas para o cadastramento das equipes de Avaliação e Acompanhamento das Medidas Terapêuticas Aplicáveis à Pessoa com Transtorno Mental em Conflito a Lei (EAP) ${ }^{16}$.

Em seguida, os textos foram relidos e foram destacadas as informações relevantes para a descrição da PNAISP no contexto da assistência em saúde mental das pessoas em conflito com a lei e a contribuição para a construção do modelo lógico. Assim, foi possível identificar os objetivos, os recursos, as atividades, os produtos, os resultados e o impacto da PNAISP. Destarte, construiu-se o modelo lógico com o propósito de sistematizar o funcionamento da PNAISP no tocante à assistência em saúde mental das pessoas em conflito com a lei.

O modelo lógico foi apresentado aos informantes-chave - cinco atores que estavam envolvidos diretamente com a execução da assistência em saúde mental das pessoas em conflito com a lei, no âmbito da Secretaria de Saúde do Estado (SESAP) e da Secretaria de Estado de Administração Penitenciária (SEAP) do Estado do Rio Grande do Norte - os quais foram entrevistados pela pesquisadora responsável, no próprio ambiente de trabalho dos mesmos, segundo agendamento prévio. Na ocasião identificaram a adequabilidade do modelo proposto para descrever a política e propuseram ajustes.

Destaca-se que, no âmbito do Estado do Rio Grande do Norte, locus deste estudo, a assistência às pessoas com transtorno mental em conflito com a lei ocorre, prioritariamente, no Hospital de Custódia e Tratamento Psiquiátrico (HCTP/ $\mathrm{RN})$. Atualmente, segundo dados fornecidos pela SEAP, possui uma população de 45 internos, que ocupam $100 \%$ das vagas disponíveis na instituição. Acrescente-se que a instituição conta com a seguinte equipe de saúde: médico psiquiatra (1), enfermeiro (2), técnico em enfermagem (16), psicólogo (1), assistente social (1).

As entrevistas ocorreram mediante o seguinte roteiro semiestruturado: Os elementos da PNAISP foram apresentados? Existem outros caminhos plausíveis para se alcançar os resultados esperados? Todos os fatores relevantes do contexto foram identificados e suas potenciais influências descritas? Se não, que outros aspectos podem ser acrescentados? Quais as dificuldades e/ou facilidades para operacionalizar o objetivo proposto?

Foram registradas em áudio, transcritas segundo anuência dos participantes e, na sequência, realizada a leitura-análise exaustiva das 
transcrições, de forma a extrair informações para readequações do modelo lógico proposto. Feitas as correções sugeridas, houve uma reunião de consenso, em que o modelo lógico foi reapresentado aos informantes-chave e validado através da Técnica de Grupo Nominal (TNG) ${ }^{17}$. À luz do modelo lógico validado, construiu-se uma matriz de critérios para avaliar a assistência às pessoas com transtornos mentais em conflito com a lei.

Posteriormente, seguiram-se os procedimentos empíricos e analíticos, de abordagem quantitativa, para validar o conteúdo da matriz de critérios. Participaram deste processo de validação experts selecionados através da análise de currículos submetidos à Plataforma Lattes, de acordo com a seguinte estratégia de busca: Modo de busca - assunto (título ou palavra-chave da produção) - "Saúde mental"; "Saúde Prisional"; nas bases - doutores e demais pesquisadores; formação acadêmica/titulação - Mestrado.

Foram selecionados 66 experts e, posteriormente, seus currículos analisados segundo adaptação dos critérios de Fehring ${ }^{18}$, sendo estabelecida uma pontuação mínima de cinco pontos para a seleção dos peritos na área do construto, identificando-se 48 experts com a pontuação estabelecida (Quadro 1). Seguiu-se com a busca pelos e-mails dos experts selecionados através de seus currículos, em sites das instituições nas quais trabalhavam, ou em artigos publicados. Acrescente-se que não foi encontrado o e-mail de quatro peritos e estes foram excluídos.
Recomenda-se um número de seis a vinte experts para compor o processo de validação de conteúdo $^{12}$, todavia, por se considerar as eventuais perdas por não respostas, enviou-se e-mail convite com a apresentação da pesquisa e seus objetivos, bem como o link que direcionava para o instrumento de coleta para os 44 experts previamente selecionados.

O instrumento de coleta foi construído por meio da ferramenta eletrônica Google Forms, sendo composto por quatro seções: 1) caracterização dos experts; 2) orientações aos experts sobre o preenchimento do formulário; 3 ) modelo lógico e matriz de critérios; e 4) 20 questões (itens) de múltipla escolha conforme as opções Adequado, Parcialmente Adequado ou Inadequado, com espaço aberto para "comentários ou sugestões para as inadequações" sobre cada item da matriz supracitada.

As questões de múltipla escolha referiam-se aos itens abordados na matriz de critérios, organizados segundo as dimensões de análise: estrutura (1-8), processo (9-14) e resultados (15-20). Salienta-se que os experts só acessavam as demais seções do formulário após assinarem o TCLE.

Os procedimentos analíticos ocorreram através de duas etapas Delphi. Na etapa Delphi 1, 16 experts avaliaram a matriz de critérios; na etapa Delphi 2, 12 preencheram o instrumento de avaliação. Os dados foram analisados a partir de estatística descritiva simples, por meio de frequências absoluta e relativa. Considerou-se válido

Quadro 1. Adaptação do sistema de pontuação de especialistas do modelo de validação de conteúdo de Fehring (1994).

\begin{tabular}{|l|c|l|c|}
\hline \multicolumn{1}{|c|}{ Critérios Fehring (1994) } & Pontos & \multicolumn{1}{c|}{ Critérios adaptados } & $\begin{array}{c}\text { Pontos } \\
\text { Adaptados }\end{array}$ \\
\hline Mestre em enfermagem & 4 & Mestre (Critério obrigatório) & 0 \\
\hline $\begin{array}{l}\text { Mestre em enfermagem - dissertação } \\
\text { com conteúdo relevante da área clínica }\end{array}$ & 1 & $\begin{array}{l}\text { Mestre com dissertação sobre saúde } \\
\text { mental e/ou saúde prisional }\end{array}$ & 2 \\
\hline $\begin{array}{l}\text { Pesquisa (com publicação) na área de } \\
\text { diagnósticos }\end{array}$ & 2 & $\begin{array}{l}\text { Pesquisa na área de Saúde Mental e/ou } \\
\text { saúde prisional }\end{array}$ & 3 \\
\hline $\begin{array}{l}\text { Artigo publicado na área de diagnósticos } \\
\text { em um periódico de referência }\end{array}$ & 2 & $\begin{array}{l}\text { Artigo publicado na área de Saúde } \\
\text { Mental e/ou prisional }\end{array}$ & 2 \\
\hline $\begin{array}{l}\text { Doutorado em diagnóstico } \\
\text { Prática clínica de pelo menos um ano } \\
\text { de duração na área de enfermagem em } \\
\text { clínica médica }\end{array}$ & 1 & $\begin{array}{l}\text { Experiência profissional na área de saúde } \\
\text { ou prisional }\end{array}$ & 4 \\
\hline $\begin{array}{l}\text { Certificado em área clínica médica com } \\
\text { comprovada prática clínica }\end{array}$ & 2 & $\begin{array}{l}\text { Certificado de especialização na área de } \\
\text { saúde mental }\end{array}$ & 2 \\
\hline Pontuação Máxima & 14 & $\begin{array}{l}\text { Pontuação Máxima } \\
\text { Fonten Saúde Mental e/ }\end{array}$ & 1 \\
\hline
\end{tabular}

Fonte: Elaborado pelos autores. 
o item que apresentasse mais de $70 \%$ de concordância entre os experts (avaliado como Adequado) e um Coeficiente de Validade de Conteúdo $(\mathrm{CVC})>0,8^{12}$.

Seguiram-se os preceitos éticos estabelecidos pela Resolução no 466/2012 do Conselho Nacional de Saúde, de modo que a proposta do estudo foi apreciada e aprovada por Comitê de Ética em Pesquisa. Obteve-se a anuência dos participantes, os quais assinaram o Termo de Consentimento Livre e Esclarecido (TCLE), tendo sido preservados seu anonimato e caráter voluntário.

\section{Resultados}

\section{I - Construção do Modelo Lógico e da Matriz de Critérios Avaliativos}

A partir dos documentos consultados e das entrevistas com os informantes-chave, elaborouse o modelo lógico da assistência às pessoas com transtornos mentais em conflito com a lei (Figura 1), que objetiva identificar os componentes, recursos, atividades e resultados esperados com a implantação do programa (produto e resultado final), além de buscar a identificação de uma cadeia causal de premissas que articule tais elementos.

Foram definidos dois componentes (político-gerencial e tático-operacional) e três subcomponentes, conforme proposto pelos especialistas consultados.

As divergências entre o modelo lógico proposto e a realidade encontrada em locus do estudo foram apontadas pelos informantes-chave e estão dispostas no Quadro 2.

Tomando por base o modelo lógico e a comparação entre o que está legalmente posto e a realidade, foram descritos os componentes, subcomponentes, dimensões de análise, além dos indicadores, bem como as diferentes técnicas de investigação que resultaram em uma matriz de critérios para avaliação. Destaca-se que a dimensão de análise foi organizada a partir da tríade proposta por Donabedian ${ }^{19}$ : estrutura, processo e resultados.

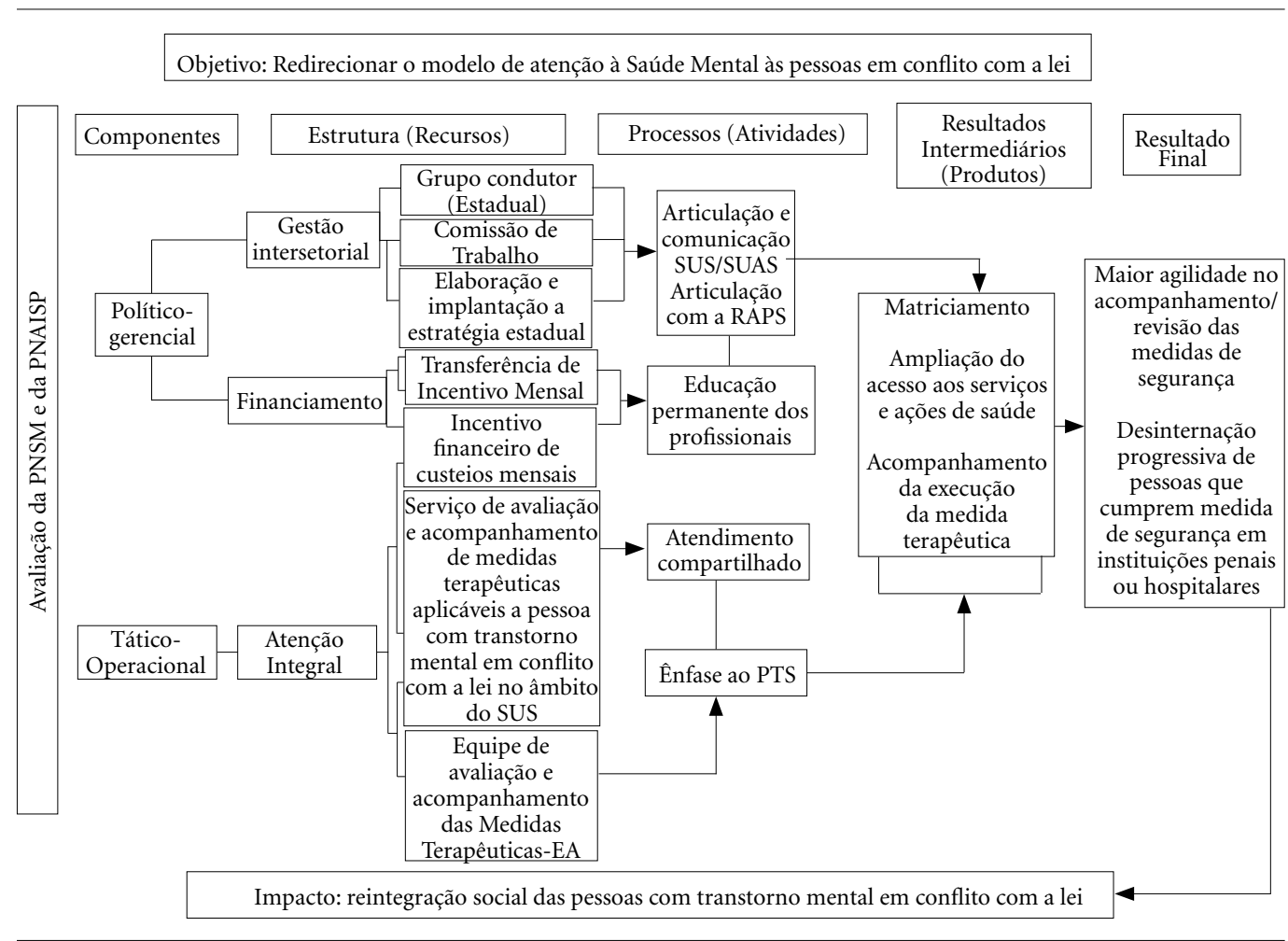

Figura 1. Modelo lógico da assistência às pessoas com transtornos mentais em conflito com a lei (PNSM/PNAISP), 2020. 
Quadro 2. Comparação entre a realidade da política e o modelo lógico de acordo com os informantes-chave, 2020.

\begin{tabular}{|c|c|}
\hline $\begin{array}{l}\text { Componente do } \\
\text { Modelo Lógico }\end{array}$ & Realidade \\
\hline Estrutura & $\begin{array}{l}\text { "As portarias são muito enxutas, deixa muita coisa sem detalhamento, a ser interpretado } \\
\text { [...], mas o grande problema é que a adesão à portaria é facultativa ao município. Então, fica } \\
\text { sob responsabilidade de quem? O Estado vai fazer tudo? Vai ser forma híbrida? Sem adesão } \\
\text { dos municípios o financiamento fica prejudicado" (Informante 1) } \\
\text { "Não há estrutura física, nem equipamentos para colocar as equipes de } \\
\text { desinstitucionalização, nem espaço físico. Aliás, não tem EAP implantada, estamos ainda } \\
\text { tentando implantar" (Informante 1) } \\
\text { "Estamos iniciando a articulação com o grupo condutor estadual, tem portaria, mas não } \\
\text { tem efetividade no trabalho ainda" (Informante 2) }\end{array}$ \\
\hline Processo & $\begin{array}{l}\text { "A grande dificuldade é a intersetorialidade, ainda é muito truncado, uma certa disputa } \\
\text { entre a justiça e a saúde, apesar das portarias serem conjuntas, não há muito diálogo entre o } \\
\text { SUS/ a SEAP/ o SUAS" (Informante 1) } \\
\text { "As ações de educação em saúde são muito pontuais, e não abordam as problemáticas de } \\
\text { forma macro" (Informante 1) } \\
\text { "A intersetorialidade é o grande problema porque o 'louco-infrator' é responsabilidade de } \\
\text { ninguém. Quem quer assumir esse papel? Quem quer dialogar sobre isso?" (Informante 3) } \\
\text { "Outro problema é que os profissionais que trabalham na Unidade Psiquiátrica de Custódia } \\
\text { e Tratamentos não são efetivos do serviço, e é em regime de plantão eventual, então } \\
\text { prejudica um pouco a questão do PTS" (Informante 4) }\end{array}$ \\
\hline Resultados & $\begin{array}{l}\text { "Uma coisa boa é que alguns CAPS têm firmado parceria para trabalhar a desinternação, } \\
\text { mas outros CAPS não estão muito preparados para receber esses internos" (Informante 1) } \\
\text { "A gente tem uma certa dificuldade de liberação dos internos, os psiquiatras não sentem } \\
\text { respaldo para sugerir a desinternação" (Informante 2) } \\
\text { "Agora que a equipe de desinstitucionalização tem um psicólogo melhorou um pouco o } \\
\text { acompanhamento e execução da medida terapêutica, porque o psiquiatra fica mais seguro, } \\
\text { porém ainda há muitas dificuldades e as mudanças são lentas" (Informante 4) }\end{array}$ \\
\hline
\end{tabular}

Fonte: Elaborado pelos autores.

\section{II - Validação da Matriz de Critérios}

Doze experts participaram da amostra final da pesquisa, com predominância de mulheres (10; 83,3\%); com média de idade de 42,6 48,9 anos; graduados em psicologia $(7 ; 58,3 \%)$ e com doutorado $(7 ; 58,3 \%)$.

A Tabela 1 mostra o resultado da avaliação dos itens da matriz de critérios, nas etapas Delphi 1 e 2. Na primeira etapa de avaliação, 11 itens foram considerados válidos em seu conteúdo e com CVC=0,89 (Tabela 1).

Realizaram-se as alterações nos itens não validados durante a etapa Delphi 1, conforme as sugestões dos experts, que foram no item 1- Acrescentar a participação comunitária na Comissão de trabalho; item 2- Subdividir em dois indicadores: elaboração e implantação das estratégias de desinstitucionalização; item 4- Detalhamento da finalidade da efetivação da transferência mental; itens 5, 8, 12, 14, 18 e 20-Acrescentar diário de campo e entrevista com usuários como técnicas de coleta para verificar os indicadores estabelecidos.
Então, submeteu-se a matriz a nova etapa de avaliação (Delphi 2). A partir dessa rodada de avaliação, todos os itens foram considerados válidos em seu conteúdo, com concordância dos experts superior a $70 \%$ e CVC $=0,93$ (Tabela 1 ). A matriz validada pelos experts pode ser vista no Quadro 3.

\section{Discussão}

A complexidade, pluralidade e intersetorialidade do campo da saúde mental denotam a necessidade de implementação de processos de avaliação nessa área, à luz de um rigor teórico-metodológico em consonância com a legislação vigente ${ }^{1,20}$.

Nesse contexto, o estudo de avaliabilidade revela-se como uma etapa fundamental, pois proporciona um ambiente favorável para avaliação, constrói entendimentos entre os envolvidos sobre a natureza e os objetivos do programa/política, busca a concordância quanto ao interesse na realização da avaliação e os possíveis usuários do 
Tabela 1. Julgamento dos itens da Matriz de Critérios na etapa Delphi 1 e Delphi 2 ( $n=16$ na Etapa Dephi 1 e n=12 na Etapa Delphi 2). Natal-RN, 2020.

\begin{tabular}{|c|c|c|c|c|c|c|c|}
\hline \multicolumn{8}{|c|}{ Etapa Delphi 1} \\
\hline \multicolumn{8}{|c|}{ Escala Likert } \\
\hline \multirow{2}{*}{ Item } & \multicolumn{2}{|c|}{ Adequado } & \multicolumn{2}{|c|}{ Parcialmente Adequado } & \multicolumn{2}{|c|}{ Inadequado } & \multirow{2}{*}{ CVC } \\
\hline & $\mathbf{n}$ & $\%$ & $\mathbf{N}$ & $\%$ & $\mathbf{n}$ & $\%$ & \\
\hline Item 1 & 11 & 68,8 & 4 & 25 & 1 & 6,3 & 0,9 \\
\hline Item 2 & 11 & 68,8 & 4 & 25 & 1 & 6,3 & 0,9 \\
\hline Item 3 & 12 & 75 & 2 & 12,5 & 2 & 13 & 0,9 \\
\hline Item 4 & 11 & 68,8 & 4 & 25 & 1 & 6,3 & 0,9 \\
\hline Item 5 & 9 & 56,3 & 7 & 43,8 & - & - & 0,9 \\
\hline Item 6 & 14 & 87,5 & 2 & 12,5 & - & - & 1 \\
\hline Item 7 & 13 & 81,3 & 3 & 18,8 & - & - & 0,9 \\
\hline Item 8 & 8 & 50 & 8 & 50 & - & - & 0,8 \\
\hline Item 9 & 12 & 75 & 4 & 25 & - & - & 0,9 \\
\hline Item 10 & 14 & 87,5 & 2 & 12,5 & - & - & 1 \\
\hline Item 11 & 16 & 100 & - & - & - & - & 1 \\
\hline Item 12 & 11 & 68,8 & 5 & 31,3 & - & - & 0,9 \\
\hline Item 13 & 13 & 81,3 & 3 & 18,8 & - & - & 0,9 \\
\hline Item 14 & 7 & 43,8 & 8 & 50 & 1 & 6,3 & 0,8 \\
\hline Item 15 & 12 & 75 & 4 & 25 & - & - & 0,9 \\
\hline Item 16 & 13 & 81,3 & 3 & 18,8 & - & - & 0,9 \\
\hline Item 17 & 13 & 81,3 & 3 & 18,8 & - & - & 0,9 \\
\hline Item 18 & 11 & 68,8 & 5 & 31,3 & - & - & 0,9 \\
\hline Item 19 & 12 & 75 & 4 & 25 & - & - & 0,9 \\
\hline Item 20 & 11 & 68,8 & 5 & 31,3 & - & - & 0,9 \\
\hline \multicolumn{8}{|c|}{ Etapa Delphi 2} \\
\hline \multicolumn{8}{|c|}{ Escala Likert } \\
\hline \multirow{2}{*}{ Item } & \multicolumn{2}{|c|}{ Adequado } & \multicolumn{2}{|c|}{ Parcialmente Adequado } & \multicolumn{2}{|c|}{ Inadequado } & \multirow{2}{*}{ CVC } \\
\hline & $\mathbf{n}$ & $\%$ & $\mathbf{N}$ & $\%$ & $\mathbf{n}$ & $\%$ & \\
\hline Item 1 & 10 & 83,3 & 1 & 8,33 & 1 & 8,3 & 0,9 \\
\hline Item 2 & 10 & 83,3 & 2 & 16,6 & - & - & 0,9 \\
\hline Item 4 & 11 & 91,7 & - & - & 1 & 8,3 & 0,9 \\
\hline Item 5 & 9 & 75 & 3 & 25 & - & - & 0,9 \\
\hline Item 8 & 10 & 83,3 & 1 & 8,33 & 1 & 8,3 & 0,9 \\
\hline Item 12 & 11 & 91,7 & 1 & 8,33 & - & - & 1 \\
\hline Item 14 & 9 & 75 & 3 & 25 & - & - & 0,9 \\
\hline Item 18 & 10 & 83,3 & 1 & 8,33 & 1 & 8,3 & 0,9 \\
\hline Item 20 & 11 & 91,7 & 1 & 8,33 & - & - & 1 \\
\hline
\end{tabular}

Fonte: Elaborado pelos autores.

estudo, e aumenta as possibilidades de uso dos resultados da avaliação ${ }^{12}$.

Identificou-se que os objetivos e metas da política, no tocante ao contexto estudado, são pouco definidos. Todavia, o desenvolvimento do modelo lógico permitiu esclarecer questões referentes ao direcionamento da PNAISP acerca da assistência às pessoas com transtornos mentais em conflito com a lei, com ênfase na desinstituciona- lização a partir das seguintes ações: implementar o Projeto Terapêutico Singular (PTS); favorecer a articulação entre os serviços do SUS e do Serviço Único de Assistência Social (SUAS); e contribuir para a realização da desinternação progressiva de pessoas que cumprem medida de segurança em instituições penais ou hospitalares ${ }^{13}$.

Não obstante, apenas a edição de normas legais não é condição suficiente para sua efetivação, 
Quadro 3. Matriz de critérios de análise, indicadores/categorias, técnicas de coleta e fontes de informação, 2020.

\begin{tabular}{|c|c|c|c|c|}
\hline Componente & Subcomponente & $\begin{array}{l}\text { Dimensão } \\
\text { de análise }\end{array}$ & Indicadores & \begin{tabular}{|c|} 
Técnica de coleta de dados \\
e informação
\end{tabular} \\
\hline & \multirow[t]{4}{*}{$\begin{array}{l}\text { Gestão } \\
\text { intersetorial }\end{array}$} & & $\begin{array}{l}\text { Implantação do Grupo condutor } \\
\text { estadual/Comissão de Trabalho } \\
\text { (com participação comunitária) }\end{array}$ & \\
\hline & & & $\begin{array}{l}\text { Elaboração de estratégia estadual } \\
\text { de desinstitucionalização }\end{array}$ & \\
\hline & & & $\begin{array}{l}\begin{array}{l}\text { Implantação de estratégia } \\
\text { estadual de desinstitucionalização }\end{array} \\
\end{array}$ & \\
\hline & & Estrutura & $\begin{array}{l}\text { Adesão dos municípios em } \\
\text { cumprimento às Portarias } \\
94,95,142 \text { de } 2014\end{array}$ & $\begin{array}{l}\text { Análise documental/ } \\
\text { Entrevista com gestores/ } \\
\text { Consulta ao SIOPS } \\
\end{array}$ \\
\hline $\begin{array}{l}\text { Político- } \\
\text { gerencial }\end{array}$ & Financiamento & & $\begin{array}{l}\text { Efetivação da transferência } \\
\text { mensal para custeio do serviço de } \\
\text { Avaliação e Acompanhamento de } \\
\text { Medidas Terapêuticas Aplicáveis } \\
\text { à Pessoa com Transtorno Mental } \\
\text { em Conflito com a Lei }\end{array}$ & \\
\hline \multirow[t]{4}{*}{$\begin{array}{l}\text { Tático- } \\
\text { operacional }\end{array}$} & \multirow[t]{2}{*}{ Atenção integral } & & Implantação das EAP & \begin{tabular}{|l|} 
Entrevista com \\
profissionais e gestores/ \\
diário de campo com \\
roteiro temático \\
\end{tabular} \\
\hline & & & Recursos humanos e materiais & \\
\hline & \multirow[t]{2}{*}{$\begin{array}{l}\text { Gestão } \\
\text { intersetorial }\end{array}$} & & $\begin{array}{l}\text { Articulação e comunicação entre } \\
\text { o SUS e//SUAS }\end{array}$ & \multirow{2}{*}{$\begin{array}{l}\text { Entrevista com } \\
\text { profissionais e gestores/ } \\
\text { diário de campo/consulta } \\
\text { ao SIOPS }\end{array}$} \\
\hline & & & Articulação com a RAPS & \\
\hline $\begin{array}{l}\text { Político- } \\
\text { gerencial }\end{array}$ & Financiamento & Processo & $\begin{array}{l}\text { Estratégias de educação } \\
\text { permanente dos profissionais do } \\
\text { SUS, SUAS e da justiça. }\end{array}$ & \\
\hline \multirow{2}{*}{$\begin{array}{l}\text { Tático- } \\
\text { operacional }\end{array}$} & \multirow[t]{2}{*}{ Atenção integral } & & Atendimento compartilhado & \multirow{2}{*}{$\begin{array}{l}\text { Análise documental } \\
\text { (prontuários/ Entrevista } \\
\text { com os usuários }\end{array}$} \\
\hline & & & Ênfase no PTS & \\
\hline \multirow[t]{2}{*}{$\begin{array}{l}\text { Tático- } \\
\text { operacional }\end{array}$} & \multirow[t]{2}{*}{ Atenção integral } & \multirow[t]{2}{*}{ Resultados } & Efetivação do matriciamento & $\begin{array}{l}\text { Entrevista com } \\
\text { profissionais e gestores } \\
\text { e análise documental } \\
\text { (Prontuários)/Entrevista } \\
\text { com os usuários }\end{array}$ \\
\hline & & & $\begin{array}{l}\text { Acompanhamento da execução } \\
\text { da medida terapêutica }\end{array}$ & \\
\hline
\end{tabular}

Fonte: Elaborado pelos autores.

pois os desdobramentos práticos destas normas devem ser concretizados em ações sistemáticas e integralizadas ${ }^{12}$.

Nesse contexto, realizar a comparação entre o modelo lógico e a realidade da política avaliada permite apreciar aspectos importantes que não são esboçados no modelo lógico, e já subsidiam o processo de avaliação subsequente ${ }^{12}$. No que concerne à estrutura, os informantes-chave apontaram a dificuldade de operacionalização do modelo lógico relacionada ao não detalhamento dos documentos oficiais, achado semelhante ao do estudo de Silva et al. ${ }^{21}$. Sobre a produção normativa no direito à saúde das pessoas com transtornos mentais em conflito com a lei, estudo de Borges e Moura ${ }^{22}$ aponta a necessidade de retificação na legislação sobre o tema que priorize a vinculação com o setor de justiça no país.

Os informantes-chave pontuaram a carência estrutural para ofertar a assistência preconizada, o que pode ser explicado tanto pelo histórico de negligência vivenciado pelo "louco infrator" ${ }^{23}$, 
como pelo aumento de internações por Medida de Segurança de usuários de álcool e outras drogas $^{24}$.

Denota-se que, além da precariedade na estrutura, o processo de reestruturação da assistência às pessoas com transtorno mental em conflito com a lei colide com a dificuldade de implementação da intersetorialidade, tanto entre os profissionais da equipe de saúde, quanto destes com relação aos profissionais da justiça ${ }^{25}$.

Os discursos pautados na hegemonia médica, em detrimento da clínica ampliada, e fundamentados no caráter punitivo das ações, em detrimento da ressocialização, dificultam a intersetorialidade de todo aquele em conflito com a lei, independente do seu estado de saúde mental ${ }^{23}$. Por sua vez, tais discursos mantêm-se, dentre outros motivos, pela insuficiência de projetos de educação continuada e permanente com foco na atenção psicossocial nos serviços ${ }^{26}$, o que contribuiu com a desarticulação entre os dispositivos da Rede de Atenção Psicossocial (RAPS) e os tribunais de justiça, e a falta de corresponsabilização pelo PTS dos sujeitos sob $\mathrm{MS}^{27}$.

A construção da matriz de critérios avaliativos elucidou a ausência de metas definidas nos documentos legais consultados, o que denota que aspectos abordados na PNAISP não são devidamente explicitados quanto à assistência às pessoas em conflito com a lei. Todavia, a matriz apresentada neste estudo pode favorecer o monitoramento sistemático por parte dos atores envolvidos com tal clientela ${ }^{12}$.

Acrescente-se que a validação do conteúdo da matriz de critérios avaliativos constitui etapa fundamental para verificar a pertinência dos itens componentes do material proposto ao construto querepresentam ${ }^{18}$.

A técnica Delphi apresenta-se como a mais apropriada para validação de instrumentos, pois, para além de buscar o consenso de profissionais com grande expertise no campo de conhecimento em tela, obtém respostas e opiniões de qualidade para uma dada questão apresentada a estes profissionais ${ }^{28}$. Quanto a sugestões dos experts na área, destaca-se a importância de incluir os usuários no processo avaliativo, pois,tal qual aponta pesquisa de Lanbecker et al. ${ }^{29}$, por vezes, a voz de pessoas com transtornos mentais é invisibilizada no cenário de pesquisas de saúde no Brasil.

Destaca-se que a validação de ferramentas de avaliação em saúde deve ser um processo frequente e contínuo, dada a dinamicidade do campo da saúde.

\section{Considerações finais}

A institucionalização da avaliação no cotidiano dos serviços configura-se como ferramenta valiosa para subsidiar processos de mudança na organização dos cenários avaliados, na medida em que proporcionam aos envolvidos ou interessados, direta ou indiretamente, condições para decidir como enfrentar e resolver problemas cotidianos.

Nessa perspectiva, a elaboração do modelo lógico e a confrontação deste com a realidade vivenciada pelos informantes-chave possibilitaram a identificação de lacunas no tocante à estrutura, ao processo e aos resultados da implementação da assistência às pessoas com transtorno mental em conflito com a lei. Ademais, a elaboração da matriz de critérios e posterior validação de seu conteúdo por experts na área podem favorecer a avaliação a que se propõe.

Ressalta-se, como uma limitação do estudo, o número reduzido de informantes-chave na execução do estudo de avaliabilidade.

Acrescente-se que o modelo lógico e a matriz de critérios construídos neste estudo podem ser estudados e revisados a qualquer momento, à medida que novas diretrizes e estratégias de implementação sejam inovadas ou que outras lentes analíticas e/ou avaliativas direcionem para a PNAISP. Recomenda-se o estabelecimento de metas exequíveis, bem como que a matriz de critérios proposta neste estudo direcione a avaliação da assistência às pessoas com transtornos mentais em conflito com a lei.

\section{Colaboradores}

LV Oliveira trabalhou na concepção do estudo, na coleta e análise dos dados e na redação final do manuscrito. AA Coelho trabalhou na concepção do estudo, na análise dos dados e na aprovação da versão a ser publicada. SAC Uchôa trabalhou na análise dos dados e na aprovação da versão final a ser publicada. PTCO Salvador trabalhou na revisão crítica do artigo e na aprovação da versão a ser publicada. CHSM Freitas trabalhou na concepção do estudo, na revisão crítica do artigo e na aprovação da versão a ser publicada. 


\section{Referências}

1. Amarante P, Nunes MO. A reforma psiquiátrica no SUS e a luta por uma sociedade sem manicômios. Cien Saude Colet 2018; 23(6):2067-2074.

2. Brasil. Lei no 10.216 , de 6 de abril de 2001. Dispõe sobre a proteção e os direitos das pessoas portadoras de transtornos mentais e redireciona o modelo assistencial em saúde mental. Diário Oficial da União 2001; 30 abr.

3. Brasil. Decreto-Lei $\mathrm{n}^{\circ} 2.848$, de 7 de dezembro de 1940 alterado pela Lei no 9.777 em 26/12/98. Código Penal. Diário Oficial da União 1940; $31 \mathrm{dez}$.

4. Cunha CC, Boarini ML. A medicina com o voto de minerva: o louco infrator. Psicol Soc 2016; 28:442-452.

5. Diniz D. A custódia e o tratamento psiquiátrico no Brasil: Censo 2011. Brasília: Letras Livres, Editora Universidade de Brasília; 2013.

6. Geopresídios. Sistema Geopresídios do Conselho Nacional de Justiça (CNJ) [Internet]. [acessado $2020 \mathrm{mar}$ 20]. Disponível em: https://www.cnj.jus.br/inspecao_ penal/mapa.php.

7. Oliveira AS, Dias FMV. Andando na contramão: o destino dos indivíduos com transtorno mental que cometem crimes no Brasil. Physis 2018; 28:e280305.

8. Brasil. Ministério da Saúde (MS). Serviço de Avaliação e Acompanhamento de Medidas Terapêuticas Aplicáveis à Pessoa com Transtorno Mental em Conflito com a Lei. Brasília: MS; 2014.

9. Brasil. Conselho Nacional de Justiça. Resolução no 113 , de 20 de abril de 2010. Dispõe sobre o procedimento relativo à execução de pena privativa de liberdade e de medida de segurança, e dá outras providências. Diário da Justiça Eletrônico 2010; 26 abr.

10. Brasil. Conselho Nacional de Política Criminal e Penitenciária. Resolução no 4, de 30 de julho de 2010. Dispõe sobre as Diretrizes Nacionais de Atenção aos Pacientes Judiciários e Execução da Medida de Segurança. Diário Oficial da União 2010; 2 ago.

11. Brasil. Portaria no 94 de 15 de janeiro de 2014. Dispõe sobre a avaliação e acompanhamento de medidas terapêuticas aplicáveis à pessoa com transtorno mental em conflito com a Lei, vinculado à Política Nacional de Atenção Integral à Saúde das Pessoas Privadas de Liberdade no Sistema Prisional. Diário Oficial da União 2014, 15 jan.

12. Pasquali L. Instrumentação psicológica: fundamentos e práticas. Porto Alegre: Artmed; 2010.

13. Mendes MFM, Cazarin G, Bezerra LCA, Dubeux LS. Avaliabilidade ou pré-avaliação de um programa. In: Samico A, Felisberto E, Figueiró AC, Frias PG, colaboradores. Avaliação em Saúde: bases conceituais e operacionais. Rio de Janeiro: MedBook; 2010. p. 57-64.

14. Brasil. Ministério da Saúde (MS). Secretaria de Atenção à Saúde. Departamento de Ações Programáticas Estratégicas. Coordenação de Saúde no Sistema Prisional. Política Nacional de Atenção Integral à Saúde das Pessoas Privadas de Liberdade no Sistema Prisional. Brasília: MS; 2014.

15. Brasil. Portaria no 95, de 14 de janeiro de 2014. Dispõe sobre o financiamento do serviço de avaliação e acompanhamento às medidas terapêuticas aplicáveis ao paciente judiciário, no âmbito do Sistema Único de Saúde (SUS). Diário Oficial da União; 2014.
16. Brasil. Portaria no 142 , de 28 de fevereiro de 2014. Estabelece normas para o cadastramento das equipes de Avaliação e Acompanhamento das Medidas Terapêuticas Aplicáveis à Pessoa com Transtorno Mental em Conflito a Lei (EAP). Diário Oficial da União; 2014.

17. Pope C, Mays N. Pesquisa Qualitativa na Atenção à Saúde. Porto Alegre: Artmed; 2009.

18. Fehring RJ. The Fehring model. In: Carrol-Johnson RM, Paquete M. Classification of nursing diagnoses: proceeding of the tenth conference. Philadelphia: $\mathrm{Li}$ ppincott Company; 1994. p. 55-62.

19. Donabedian A. Basic approaches to assessment: structure, process and outcome. In: Donabedian A. Explorations in quality assessment and monitoring. Michigan: Health Administration Press; 1980. p. 77-125.

20. Bessa MS, Araújo Filho JD, Azevedo DM, Salvetti MG, Torres GV. Avaliação de Resultados da Assistência em Saúde Mental por Usuários. Rev Bras Cien Saude 2019; 23:89-96.

21. Silva RN, Guarda FRB, Hallal PC, Martelli PJL. Avaliabilidade do Programa Academia da Saúde no Município do Recife, Pernambuco, Brasil. Cad Saude Publica 2017; 33:e00159415.

22. Borges AAB, Moura LM. Análise da produção normativa no direito à saúde dos inimputáveis - doentes mentais infratores (2011-2014). Cad Ibero-Amer Dir Sanit 2015; 4:83-102.

23. Soares Filho MM, Bueno PMMG. Demografia, vulnerabilidades e direito à saúde da população prisional brasileira. Cien Saude Colet 2016; 21(7):1999-2010.

24. Lhacer PMV. Transinstitucionalização: Caminhos e Descaminhos na Dinâmica de Internações e Desinternações de Pessoas com Transtorno Mental em Conflito com a Lei no Estado de São Paulo [tese]. São Paulo: Universidade de São Paulo; 2019.

25. Oliveira TTSS, Abrici EP, Santos MA. Estrutura e funcionamento de uma equipe de saúde mental de Trieste na perspectiva de seus integrantes: um estudo qualitativo. Psicol Pesq 2018; 12(2):24-35.

26. Ferreira TPS, Sampaio J, Souza ACN, Oliveira DL, Gomes LB. Produção do cuidado em Saúde Mental: desafios para além dos muros institucionais. Interface (Botucatu) 2017; 21(61):373-384.

27. Cortez PA, Souza MVR, Oliveira LFA. Princípios de uma política alternativa aos manicômios judiciais. Saude Soc 2018; 27(4):1206-1217.

28. Scarparo AF, Laus AM, Azevedo ALCS, Freitas MRI, Gabriel CS, Chaves LDP. Reflexões sobre a técnica delphi em pesquisa na enfermagem. Rev Rene 2012; 13(1):242-251.

29. Langbecker A, Castellanos MEP, Neves RF, Catalan -Matamoros D. Journal coverage on issues of interest to Brazilian Collective Health: a literature review. Interface (Botucatu) 2019; 23:e1800095.

Artigo apresentado em 06/06/2020

Aprovado em 03/09/2020

Versão final apresentada em 05/09/2020

Editores-chefes: Romeu Gomes, Antônio Augusto Moura da Silva 